\title{
DYNAMICS BASED CONTROL OF VERTICALLY ARTICULATED MANIPULATORS
}

\author{
M. B. Leahy Jr. \\ Air Force Institute of Technology \\ Department of Electrical and Computer Engineering \\ WPAFB OH 45433
}

\begin{abstract}
The relationship between tracking accuracy and the model employed in dynamics based control, of a vertically articulated manipulator, has been experimentally evaluated. High gear ratios reduce the payload sensitivity and computational complexity of the system dynamics but do not eliminate the performance enhancement from accurate compensation of dominant dynamics. Robot controllers must compensate for changes in dominant drive system, link, and payload dynamics to achieve high speed trajectory tracking accuracy. Feedforward dynamic compensation reduced the peak trajectory tracking errors of an independent joint PD controller by a factor of three. Knowledge of payload mass and centroid allowed feedforward dynamic compensation to overcome the disturbances produced by unmodeled payload dynamics. The combination of high sample rates, constant PD gains, and adaptive feedforward dynamic compensation will produce a control algorithm with excellent payload independent, peak and final error
\end{abstract}

\section{INTRODUCTION}

Restrictions on maximum load and speed of industrial robots are a function of control law limitations and not those of the manipulator design [5]. Velocity and acceleration limits are artifically imposed so that the disturbance input to the robot controller from unmodeled dynamics is bounded. Under these conditions a conventional independent joint PD control law can be tuned to reach the desired repeatibility and stability specifications, but the maximum capabilities of the robot can not be exploited. Improvements in controller performance would increase industrial manipulator productivity and range of applications without costly redesign of drive system components.

The performance improvement potential of dynamic compensation has been experimentally evaluated on direct $[1,7]$, hydraulic [4] and gear driven manipulators [14]. Direct drive manipulators provide an ideal test platform for dynamic based control techniques because the dominant dynamics can be obtained from a standard Newton-Euler formulation. The drive systems of vertically articulated manipulators must supply high torque amplification. The ability to neglect motor dynamics and create an ideal environment is destroyed by the introduction of those high ratio geartrains. The role of dynamic compensation in a nonideal environment is unclear due to the properties of actuator inertia, viscous and static friction, backlash, and other nonlinear geartrain effects that complicate the dynamic equations. Initial evaluations of gear driven manipulator dynamic compensation were clouded by incomplete modeling of geartrain dynamics and a trajectory containing jerk constraints $[14,16]$.

If dynamic compensation could eliminate the performance restrictions of current controllers the productivity and range of applications of geared manipulators would be increased. The trajectory tracking accuracy of algorithms that employ dynamic compensation is dependent on accurate knowledge of the link and payload dynamics. The disturbances produced by unmodeled payload dynamics can seriously degrade tracking performance [8]. Craig, Hsu, and Sastry proposed an parameter-adaptive control scheme that combined a set of adaptive laws with a computedtorque algorithm to reduce the disturbances from payload uncertainty [8]. Tzes and Yurkovich proposed dividing the dynamics based control law into two components based on sensitivity analysis applied to the complete dynamic model of the manipulator [20]. The payload independent dynamics would provide a nominal control input, that could be calculated off-line, and the second component would provide payload dependent torques based on periodic estimates of payload mass [20]. Additional load sensitivity analysis has shown that the disturbances produced by variations in individual joint dynamics due to loading may only depend on a subset of the payloads inertial parameters [3]. Asada and Hara proposed eliminating those disturbances by adapting the feedforward compensation to that subset of payload dynamics [3]. The performance of their adaptive feedforward technique has been experimentally evaluated on a three degree of freedom(DOF) SCARA direct drive arm [3]. Vertically articulated manipulators require drive systems with high torque amplification. The high gear ratios of those drive units, reduce but do not eliminate, the sensitivity of joint dynamics to payload variations. mine:

The objectives of this research were to experimentally deter-

1. the degree of improvement in the unloaded high speed tracking accuracy from compensating for specific manipulator dynamics by feedforward and computed-torque controllers,

2. the sensitivity to payload variations, and

3. whether adaption of dynamics based controllers was necessary to maintain high speed trajectory tracking accuracy,

for vertically articulated manipulators. Those objectives were meet by systematically evaluating the high speed trajectory tracking efficacy of a PUMA-560. The PUMA is an appropriate case study because; the gear ratios are high [2], the existing controller severly limits productivity [5], the inertial dynamics have been extensively studied [19,2], drive system dynamics have been experimentally determined [13], and a modified control system which allows torque to be specified has been developed [15]. 
Therefore. results from PUMA research have implications on controller design for the whole class of gear driven articulated manipulators. The results of that research are presented in the following sequence. A complete model of the PUMA control system model is documented in section two. Section three presents an analysis of the effects of dynamic compensation on unloaded manipulator trajectory tracking accuracy. In section four the results of section three are extended in an analysis of the impact of uncertain payload dynamics on controller performance. Conclusions and on-going research are in section five.

\section{MANIPULATOR MODEL}

In order to accurately specify the response of even a simple PD control law the designer must have an accurate knowledge of the system dynamics. Each link of the PUMA manipulator can then be modeled as:

$$
n^{2} J_{m} \ddot{q}+n^{2} B_{m} \dot{q}+\tau_{l}+\tau_{f}=n \tau_{m}=n K_{c} C
$$

Where:

$J_{m}=$ inertia of the motor actuator

$B_{m}=$ viscous friction of the motor

$\tau_{f}=$ static friction torque

$K_{\mathrm{c}}=$ count to torque conversion

$n=$ gear ratio (motor/link)

$C=$ counts

$\tau_{m}=$ torque supplied to the motor

$q, \dot{q}, \ddot{q}=$ position, velocity and acceleration of the link

$\tau_{l}=$ motor load torque commonly expresssed as: $\sum_{j=1}^{6} D_{i j} \ddot{q}_{j}+$ $\sum_{k=1}^{6} \sum_{l=k}^{6} C_{j k l}(q) \dot{q}_{k} \dot{q}_{l}+g(q)$ where the terms represent; inertial forces, Coriolis and centrifugal forces, and gravitational torque respectively.

Motor torque is controlled by sending a count value to the PUMA hardware controller which is converted to an analog motor current signal. The torque to count conversion values are from [2]. The values of $J_{m}$ are from Tarn [19]. Results from Tarn's research also provide the PUMA inertial parameters employed by the symbolically reduced Lagrange-Euler formulation to determine $\eta_{l}[19]$. Static friction was compensated for by an additive velocity dependent switching function successfully employed in earlier studies [14]. The $B_{m}$ values were experimentally determined for each joint, and our assumption that PUMA dynamics can be represented as a second order model was validated [13].

\section{CONSTANT PAYLOAD ANALYSIS}

A foundation for future research was developed by first analyzing the impact of dynamic compensation on the PUMA-560 in the unloaded configuration, where the dynamic structure and inertial parameters are known $\{19,13]$.

\subsection{METHOD OF APPROACH}

To verify our hypothesis, that the existing PUMA controller was inadequate for high speed trajectory tracking, the nominal PUMA controller was commanded to track the test trajectories. The test trajectories are identical to those employed in previous research [14]. The shape of the resultant position error profile was indicative of a stiff PD loop. As Table 2 shows, the original controller produced minimal final errors but allowed peak error to exceed three degrees [13]. Therefore, research efforts concen- trated on how to improve trajectory tracking accuracy without adversely impacting steady state response.

Previous research has identified the significant role of static friction and gravity compensation in PUMA manipulator dynamics $[13,14]$. Therefore an algorithm that compensates for those forces was designed to serve as a performance baseline. Then individual inertial components were added into the feedforward path. Finally two computed-torque algorithms $[1,7]$ were evaluated to ascertain the effects of self inertia variation and error coupling on tracking efficacy. By defining error as desired minus actual joint position $e=q_{d}-q_{a}, B_{e f f}=n^{2} B_{m}, J_{\text {eff }}=n^{2} J_{m}+D_{i i}$, and $\tau_{\text {eff }}=n \tau_{m}-\tau_{f}$ the joint torque equations for the evaluated algorithms can be written as:

a. Individual joint PD with static friction and gravity compensation

$\tau_{e f f}=K_{v} \dot{e}+K_{p} e+g\left(q_{a}\right)$

b. Individual joint PD with complete single joint feedforward $r_{e f f}=K_{v} \dot{e}+K_{p} e+g\left(q_{a}\right)+J_{e f f} \ddot{q}_{d}+B_{e f f} \dot{q}_{d}$

c. Individual joint PD with complete feedforward; $D_{j j}=J_{e f f}$ $\tau_{e f f}=K_{v} \dot{e}+K_{\mathrm{p}} e+g\left(q_{a}\right)+\sum_{j=1}^{\beta} D_{i j} \ddot{q}_{d}+B_{e f f} \dot{q}_{d}$

d. Computed-torque with diagonal inertia $\tau_{e f f}=J_{e f f}\left(\ddot{q}_{d}+2 \zeta \omega_{n} \dot{e}+\omega_{n}^{2} e\right)+B_{e f f} \dot{q}_{a}+g\left(q_{a}\right)$

e. Computed-torque with full inertia

$$
\tau_{e f f}=\sum_{j=1}^{6} D_{i j}\left(\ddot{q}_{d}+2 \zeta \omega_{n} \dot{e}+\omega_{n}^{2} e\right)+B_{e f f} \dot{q}_{a}+g\left(q_{a}\right)
$$

For a constant $J_{e f f}$ and $B_{e f f}, K_{v}$ and $K_{p}$ can be calculated to provide the desired system response [13]. However, only joints 46 , where actuator inertia dominates, have a constant $J_{\text {eff }}$ when the manipulator is unloaded. If $J_{e f f}$ is not a constant, Koren las demonstrated that best PD performance is achieved when the gains are determined utilizing the minimum $J_{e f f}$ value [9]. The $K_{v}$ and $K_{p}$ values were calculated using the minimum $J_{\text {eff }}$ values shown in Table 1 and the requirement that the system response be critically damped with $\omega_{n}=15$ for large joints and $\omega_{n}=20$ for small joints.

\subsection{EXPERIMENTAL EVALUATION}

Each of the algorithms was experimentally evaluated on a PUMA560 operating under a modified version of the RAL Hierarchical Control System [15]. Analysis of the resultant error profiles has clearly illustrated the performance improvement potential from compensating for specific system model components. Peak and final error values for test trajectory zero are in Table 2. Figures 1 and 2 are representative position error profiles. Additional evaluation data is cataloged in [13].

Manipulator model components can be segregated into two groups; motion independent and motion dependent. Motion independent dynamics present a constant disturbance to the PD controller. Elimination of those disturbances improves controller performance over the whole trajectory decreasing both peak and final position errors, independent of speed [13]. For high speed trajectory tracking the forces of motion dependent dynamics become significant. The components of articulated manipulator motion dependent dynamics are self inertia, inertial coupling and viscous friction.

In our PUMA case study, compensation of motion dependent dynamics caused an appreciable improvement in trajectory tracking accuracy for all joints except the second. Joint 2 velocity and acceleration were fifty percent of the other large joint values. Low velocity and acceleration limits the disturbances caused by 
the motion dependent dynamics. Under those conditions gravity dominates joint dynamics. Therefore gravity compensation dominated tracking accuracy improvement.

The common assumption that high gear ratios negate the influence of inertial coupling on overall system torque [18] is false. Conclusions about the significance of specific dynamic components can only be justified after analysis of the individual robot dynamics. The actuator dominance of small link inertial dynamics negates the effects of inertial coupling. However, PUMA large link inertial coupling terms are a significant component of motion dependent dynamics. Without inertial coupling information, feedfor ward controllers produce large joint error profiles that lead and then lag, just as perdicted by simulation studies that incompletely model the motor dynamics [16]. For the trajectories used the inclusion of inertial coupling in the feedforward loop reduces the feedforward torque, decreasing the magnitude of the lead and lag peaks.

\subsection{DISCUSSION}

Ideally the frequency response, open loop gain, and sample rate of the PD loop employed in these evaluations should have been equivalent to the existing controller. The nominal PUMA controller has a 0.875 us sample rate. Because of the computation limitations of the RHCS control computer, the dynamics employed in the computed-torque with full inertia algorithm(e) could only be calculated every $14 \mathrm{~ms}$. To eliminate the sample rate variable from our evaluations all algorithms used $14 \mathrm{~ms}$ san pling times. The $14 \mathrm{~ms}$ sample rate did not prevent dynamic compensation from reducing trajectory tracking accuracy to un der 0.75 degrees, but the evaluated algorithms were unable to consistently drive final error values down to the level of the existing controller. The inferior final errors can be directly at tributed to the low sample rate. Final error is dependent on the open loop gain, stiffness, of the PD loop. In electromechanica systems high sample rates are necessary if stiff PD loops are to employed without producing instability. An increase in sample rate will allow the increased stiffness required for superior final end point control without reducing the performance improvement from dynamic compensation [6].

In theory the computed-torque algorithm will produce a set of independent error equations that asymptotically drive the error to zero. Any variation from a zero final error is due to inaccurate modeling and finite robot trajectories. However, the derivation of the computed-torque algorithm doesn't consider the impact of error coupling on peak error values. Unless individual joint errors remain small, the computed-torque algorithm can produce peak errors inferior to those of a feedforward algorithm. As Figure 1 shows, algorithm e significantly decreases the manipulator's ability to track test trajectory zero.

At the start of test trajectory zero all links have a positive desired acceleration. Joint 1 's inability to track the desired position trajectory produces a large positive error in position and velocity. The PD loop attempts to reduce that error by increasing the command input. The computed-torque algorithm sums the $\mathrm{PD}$ loop input with the desired acceleration to produce a new joint acceleration value. That acceleration value is then used to decouple the joints by compensating for inertial coupling. But the coupled acceleration value is incorrect and the other joints over/undercompensate for joint interactions, reducing the controller's trajectory tracking ability. Eventually the PD loop val- ues for each joint become large enough to overcome the incorrect compensation and the algorithm begins to track again.

Experimental evaluations on the MIT direct drive arm have shown that as sampling rate is decreased the difference between computed-torque and feedforward control is blurred [1]. Use of feedforward dynamic compensation would eliminate the potential hazards of error coupling inherent in computed-torque controllers without a performance penalty.

\section{VARIABLE PAYLOAD ANALYSIS}

The results of the previous section demonstrated the ability of dynamic compensation to improve unloaded manipulator performance. Future robotic research applications, such as robotic telepresence, require a vertically articulated manipulator with the ability to duplicate the human's ability to quickly and accurately move variable loads. Further studies of dynamics based control of direct drive manipulators identified a severe performance degradation due to disturbances from unmodeled payload dynamics [3]. The impact of payload variations on dynamics based control of vertically articulated manipulators must be determined before the controller can be utilized in an uncertain load environment.

\subsection{METHOD OF APPROACH}

The performance of the feedforward compensated PD (FCPD) algorithm described by (c) was reevaluated under variable payloads to determine whether adaption of feedforward compensation is necessary to preserve tracking accuracy. A goal of this research was to determine; the role of individual dynamic components in causing that degradation, and the minimum subset of payload dynamics necessary to restore tracking accuracy by adaptive feedforward compensation.

The payload dynamics are determined by three factors; radius of gyration $\left(k_{x x}^{2}, k_{y y}^{2}, k_{z z}^{2}\right)$, mass, and mass centroid. To determine the influence of those terms on joint dynamics two loads were utilized. The test loads were rigidly attached to link 6 . Both loads are symmetric, with an jdentical mass that meets the maximum load specification for the PUMA. The main difference between the loads is their radius of gyration. Load one was a steel cylinder with a $70 \mathrm{~mm}$ diameter and corresponding small radius of gyration. Load two was steel bar with a $25 \mathrm{~mm}$ diameter and length of $500 \mathrm{~mm}$ that was attached in line with the $\mathrm{Y}$ axis of joint 6 by a $50 \mathrm{~mm}$ high aluminum mounting bracket. Load two has a high $k_{x x}^{2}$ and $k_{z z}^{2}$.

Systematic analysis of the dynamic compensation torques, produced by the arm models employed in the feedforward loop, identified the impact of individual dynamic components on performance degradation [11]. Once the effect of loading on system dynamics was known, the level of modeling needed to cancel that effect was determined and experimentally verified.

Payloads also effect the feedback loop's ability to meet the desired performance specifications. The FCPD algorithm employs PD feedback loops around each joint. The position and velocity gains used in those loops were based on the minimal joint inertia over the unloaded manipulators entire range of motion. Any variation in self inertia decreases the damping ratio $(\zeta)$ and natural frequency $\left(\omega_{n}\right)$ of the feedback controller as shown below [9]:

$$
\begin{aligned}
& \zeta_{\text {actual }}=\zeta \sqrt{J_{\min } / J_{\text {actual }}} \\
& \omega_{n a}=\omega_{n} \sqrt{J_{\min } / J_{\text {actual }}}
\end{aligned}
$$


In order to avoid underdamped response for all arm configurations $\zeta$ must be modified so that:

$$
\zeta=\zeta_{\min } \sqrt{J_{\max } / J_{\min }}
$$

where $\zeta_{\min } \geq 1.0$. The price of this compromise is a degradation in controller performance whenever the loading, self inertia, is reduced. Maximum degradation occurs when the robot is unloaded. The reduction in arm performance depends on the variations that payload induces in individual joint self inertias. If the difference between maximum and minimum self inertia values is small, the degradation in unloaded performance from designing for maximum load, and thus performance penalty from utilization of constant gains, will be slight.

For an unloaded PUMA, the self inertia and therefore $\zeta$ and $\omega_{n}$ are constant over the entire range of motion for joints 3-6 Loading increases the self inertia of all the links and causes joint 3 and 4 values to vary over a given trajectory. Therefore, joint 3-6 response is no longer constant and must be redesigned to insure overdamped response. A new set of feedback gains was calculated using $\zeta$ values determined from $(4)$ with $\zeta_{\min }=1.0$ and $J_{\max }$ from Table 1 [11]. The performance degradation from incorporation of those gains into the FCPD algorithm was then experimentally determined.

\subsection{VARIATIONS IN DYNAMIC COMPENSA- TION}

Figures 3-5 illustrate both the performance degradation from unmodeled payload dynamics and the improvement from incorporating selective knowledge of payload dynamics into the feedforward compensator.

For links 2-5 the variation in compensation torques is dominated by gravity. Analysis of the symbolic dynamic equations proves that the gravity equations depend on payload mass and centroid. For joints 2 and 3 , the payload centroid variations have a minimum impact on gravitational dynamics. Payload mass and centroid are also the only load parameters that are reflected into large link inertial dynamics. Once again the effect of centroid information on dynamic component calculations is minimal. Therefore, knowledge of the payload mass is all the dynamics information required to accurately compensate for the effect of payload on large joint tracking accuracy.

The analysis of the previous section concluded that for an unloaded PUMA manipulator, the small links can be modeled as a series of uncoupled, constant inertia, second order systems. The low inertias and high gear ratios of the small links negates the effects of inertial coupling and gravity allowing motor dynamics to dominate system dynamics. However, those conclusions are no longer valid in the presence of heavy payloads. Under heavy payload configurations, both gravity and inertial coupling become significant forces that must be accounted for to achieve maximum controller performance. A $2.3 \mathrm{~kg}$ payload, the maximum allowable for a PUMA, increases the inertial coupling between joint 5 and the large links by a factor of 100 , while increasing self inertia by only 50 percent. Inertial coupling terms are now the same order of magnitude as the self inertia and produce a noticeable portion of overall joint torque. The magnitude of joint 5 's inertial coupling terms depends on payload; mass, centroid, and $\mathrm{x}$ axis radius of gyration, but variations in $k_{x x}^{2}$ have a minimal impact on overall output torque.
Like joint 5 , changes in joint 4 gravitational forces depend only on payload mass and centroid. However, joint 4's inertial coupling forces are heavily dependent on both $k_{x x}^{2}$ and $k_{z z}^{2}$. Payloads with small radius of gyration produce a negligible change in large link inertial coupling terms. Small gyration payloads like load 1 , increase coupling between joint 4 and 6 by a factor of 100 , but that is still insignificant compared to joint self inertia. For a payload with high $k_{z z}^{2}$ and/or $k_{x x}^{2}$ the effects of inertial coupling on joint 4 dynamics become significant.

The combined link and load dynamics of joint 6 depend only on mass and $k_{z z}^{2}$. For small $k_{z z}^{2}$ the inertial coupling terms are negligible, becoming significant only for large $k_{z z}^{2}$. Therefore, changes in small link dynamics produced by addition of a small inertia payload can be accurately modeled by knowledge of mass and centroid. However for complete compensation of the effect of payloads with large radius of gyration, on joints 4 and $6 \mathrm{dy}$ namics, $k_{z z}^{2}$ and $k_{x x}^{2}$ must be known

\subsection{VARIATIONS IN FEEDBACK RESPONSE}

The changes in PUMA feedback loop damping ratios necessary to guarantee overdamped response for all payload configurations are shown in Table 1 . The small increases in joint self inertia produced by application of the maximum payload keep all $\zeta$ values under 1.5. The degradation in unloaded performance that results from utlilization of the slightly overdamped $\zeta$ values is negligible. The largest variation in joint 3-6 absolute position error caused by tuning the controller for maximum load was 0.001 radians or 0.057 degrees [11]. The small variations in peak errors are directly related to the PUMA's high gear ratios.

For manipulators with high gear ratios the utilization of a constant $\mathrm{PD}$ loop in a dynamics based algorithm imposes no noticeable performance penalty. High gear ratios reduce the payload sensitivity of self inertia values [3], especially for the small links. Adaptive control is more appropriate for direct drive arms where the self inertial variations are not masked by high actuator inertia values. Under those circumstances replacement of the FCPD algorithm by an algorithm whose feedback loop adapts to changes in self inertia, such as the adaptive controller proposed by Craig [8] may be warranted.

\subsection{DISCUSSION}

PUMA results are consistent with those from Asada and Hara's [3] study of direct drive manipulator load sensitivity. In both cases; adapting the feedforward compensation to variations in load parameters greatly increased the tracking accuracy, feedback gain adaption had little effect on accuracy, and variations in large link dynamics could be accurately approximated by knowledge of mass alone. Therefore, the need to account for payload radius of gyration and centroid in industrial manipulator small link feedforward adaption should extend to direct drive arms.

A symbolic on-line representation of arm dynamics would allow the controller to quicky adapt to changing payloads. The computational capability of current microprocessors is sufficient to implement an on-line version of the FCPD algorithm [17]. The additional calculations necessary to model small link inertial coupling and gravity increases the computational burden from calculation of feedforward compensation by 42 and 18 floating point multiplies and additions to 107 and 64 total [12]. Recalculation of the payload dependent constants used by the symbolic equations only takes 25 floating point multiplies and 19 floating points addi- 
tions each time the load is changed [12]. The symbolic reduction techniques applied to the PUMA could reduce the computational burden of other articulated manipulators to similar low levels.

\section{CONCLUSIONS}

The performance improvement capability of industrial manipulator dynamic compensation has been clearly demonstrated. In the unloaded PUMA case study feedforward dynamic compensation was able to reduce the peak trajectory tracking error of all joints to under 0.75 degrees. Vertically articulated manipulators with high torque amplification drive systems:

- require accurate modeling of drive system dynamics,

- reduce the computational complexity of the system dynamics by negating the impact of Coriolis and centrifugal forces,

- reduce payload sensitivity of system dynamics, but

- do not eliminate the requirement for accurate modelling of gravity and inertial coupling.

For manipulators with either direct $[1,7]$ or high torque amplification drive systems:

- to achieve maximum trajectory tracking accuracy, the dominant system dynamics must be compensated for

- alterations in system dynamics produced by payload variations can be compensated for by adaptive feedforward compensation.

Tracking performance of dynamics based algorithms will degrade in the presence of disturbances generated by an unmodeled payload, but knowledge of complete payload dynamics is not required to regain accuracy. Knowledge of payload mass allows the feedforward compensator to adapt to changes in large link dynamics due to payload variations. Small link adaption requires mass and centroid information. Only payloads with large radii of gyration require complete knowledge of load dynamics required to eliminate the disturbances produced by loading. Adapting gains to changes in self inertia would have a minimal impact on the trajectory tracking accuracy of revolute industrial manipulator controlled by dynamics based algorithms.

Although dynamic compensation relaxes the sample rate requirements for accurate tracking, a stiff $P D$ loop is still required for minimal final errors. Because stiff PD loops require a high sample rate, an overall improvement in controller performance can only be obtained if all the dominant dynamics of the manipulator system are accounted for and high sample rates are available. The combination of high sample rates, fixed gain PD feedback, and adaptive feedforward dynamic compensation will produce a control algorithm with excellent payload independent, peak and final errors.

The long term goal of this research is the development and evaluation of control techniques that will provide manipulators with the range, speed, and adaptibility of a human arm. Current research centers around developing additional techniques for adaption of feedforward dynamic compensation.

\section{References}

[1] An, C. H., Atkeson, C. G., Griffiths J. D., and Hollerbach, J. M., "Experimental Evaluation of Feedforward and Computed Torque Control", Proc. of the Int. Conf. on Robotics and Automation, pp. 165-8 Raleigh, NC, Marcle 31-April 3, 1987.
(2) Armstrong, B., Khatib, O., and Burdick J.,"The Explicit Dynamic Model and Inertial Parameters of the PUMA 560 Arm, ${ }^{n}$ Proc. of the IEEE Int. Conf. on Robotics and Automation, pp. 510-8, San Francisco, Ca. April 1986.

[3] Asada, H., and Hara, L.," Load Sensivity Analysis and Adaptive Control of a Direct Drive Arm," Proc of the 1986 ACC, pp. 799. 805 , Seattle, WA, June 1986.

[4] Craig, J. J., Hsu, P., and Sastry, S. S., "Adaptive Control of Mechanical Manipulators," The Int Journal of Robotics Research, Vol. 6, No. 2, pp. 16-28, Summer 1987.

[5] Egeland, O., "Cartesian Control of a Hydraulic Redundant Manipulator," "Proc. of the Int. Conf. on Robotics and Automation, pp. 2081-7, Raleigh, NC, March 31-April 3, 1987.

[6] Goor, R. M., "A New Approach to Minimum Time Robot Control," ASME Winter Annual Meeting; Robotics and Manufacturing Automation, PED-Vol 15, pp. 1-11, Maimi Beach, Nov. 17-22, 1985.

[7] Khosla, P. K., "Choosing Sampling Rates for Robot Control," Proc. of the Int. Conf. on Robotics and Automation, pp. 169-74 Raleigh, NC, March 31-April 31987.

[8] Khosla, P. K. and Kanada, T., "Experimental Evaluation of the Feedforward Compensation and Computed-Torque Control Schemes," Proc of the American Control Conf., pp. 790-8, Seattle, WA, June 1986.

[9] Koren, Y., Robotics for Engineers,McGraw-Hill, 1985.

[10] Kubo, T., Anwar, G. and Tomizuka, M., "Application of Nonlinear Friction Compensation to Robot Arm Control," Proc. of the IEEE Int. Conf. on Robotics and Automation, pp. 722-7, San Francisco, Ca. April 1986.

[11] Leahy, M.B. Jr., "Compensation of Industrial Manipulator Dynamics, Part 2" RAL Technical Report, No.107, RPI, December 1987, submitted to the Int Journal of Robotics Research.

[12] Lealy, M. B. Jr., Nugent, L. M., Valavanis, K. P. and Saridis, G. N., "Efficient Dynamics for A PUMA-600," Proc. of the IEEE Int. Conf. on Robotics and Automation, pp. 519-24, San Francisco, C.A, April 1986.

[13] Leahy, M.B. Jr., and Saridis, G. N., "Compensation of Industrial Manipulator Dynamics, Part 1" RAL Techuical Report, No.101, RPI, September 1987, submitted to the Int Journal of Robotics Research.

[14] Leahy, M. B. Jr., and Saridis, G. N., "Compensation of Unmodeled PUMA Manipulator Dynamics," Proc. of the Int. Conf. on Robotics and Automation, pp. 151-6 Raleigh, NC, March 31-April $3,1987$.

[15] Leahy, M. B. Jr., and Saridis, G. N., "The RAL Hierarclical Control System," Proc. of the Int. Conf. on Robotics and Automation, pp. 407-11, San Francisco, CA, April 1986.

[16] Leahy, M. B. Jr., Valavanis, K. P. and Saridis, G. N., "The Effects of Dynamics Models on Robot Control," Proc. of the Int. Conf. on Robotics and Automation, pp. 49-54, San Francisco, CA, April 1986.

[17] Liu, C. H., and Chen, Y. M., "Multi-Microprocessor-Based Cartesian-Space Control Technique for a Mechanical Manipulator," IEEE Journal of Robotics and Automation, Vol RA-2, No. 2, pp. 110-5, June 1986 .

[18] Sweet, L. M., and Good , M. C., "Redefinition of the Robot Motion-Control Problem," IEEE Control Systems Magazine, Vol. 5. No. 3, 1985.

[19] Tarn, T. J., Bejczy, A. K., Hau S., and Yun X., "Inertia Paraneters of PUMA 560 Robot Arm," Robotics Laboratory Report SSM-RL-85-01, Washington University, St. Louis, Mo., September 1985 .

[20] Tres, A., and Yurkovich, S., "A Sensitivity Analysis Approach to Control of Manipulators with Unknown Load," Proc. of the Int. Conf. on Robotics and Automation, pp. pp. 496-502, Raleigh, NC, March 31-April 31987. 
TABLE 1

Variations in Self Inertia and $\zeta$ Magnitudes

\begin{tabular}{||c|c|c|c|c|c|c||}
\hline \multirow{2}{*}{ Joint } & $n^{3} J_{m}\left(\mathrm{kgm}^{2}\right)$ & \multicolumn{3}{|c|}{$J_{e f f}\left(\mathrm{kgm}^{2}\right)$} & \multicolumn{2}{c||}{$\zeta$} \\
\cline { 3 - 7 } & & $\max$ & unload max & min & unloaded & loaded \\
\hline 1 & 0.77 & 5.2 & 4.6 & 2.5 & 1.36 & 1.44 \\
\hline 2 & 2.36 & 8.0 & 5.9 & 5.2 & 1.06 & 1.24 \\
\hline 3 & 0.58 & 0.96 & 0.96 & 1.60 & 1.00 & 1.30 \\
\hline 4 & 0.18 & 0.25 & 0.18 & 0.18 & 1.00 & 1.17 \\
\hline 5 & 0.15 & 0.22 & 0.15 & 0.15 & 1.00 & 1.20 \\
\hline 6 & 0.18 & 0.22 & 0.18 & 0.18 & 1.00 & 1.10 \\
\hline
\end{tabular}

TABLE 2

Unloaded PUMA Tracking Error(rads)

\begin{tabular}{|c|c|c|c|c|c|c|}
\hline \multirow{2}{*}{$\begin{array}{c}\text { Algorithm } \\
\text { Letter }\end{array}$} & \multicolumn{6}{|c|}{ Joint } \\
\hline & 1 & 2 & 3 & 4 & 5 & 6 \\
\hline \multicolumn{7}{|c|}{ Peak Errors over Test Trajectory 0} \\
\hline$\overline{\text { PUMA }}$ & 0.0640 & 0.0315 & $\overline{0.0644}$ & 0.0637 & 0.0645 & 0.0652 \\
\hline $\mathbf{a}$ & 0.0272 & 0.0129 & 0.0379 & 0.0190 & 0.0230 & 0.0297 \\
\hline $\mathrm{b}$ & 0.0161 & 0.0180 & 0.0063 & 0.0065 & 0.0063 & 0.0090 \\
\hline c & 0.0104 & 0.0108 & 0.0056 & 0.0063 & 0.0063 & 0.0091 \\
\hline d & 0.0132 & 0.0174 & 0.0060 & 0.0059 & 0.0063 & 0.0091 \\
\hline e & 0.0354 & 0.0290 & 0.0207 & 0.0061 & 0.0063 & 0.0092 \\
\hline \multicolumn{7}{|c|}{ Final Errors over Test Trajectory 0} \\
\hline PUMA & 0.0010 & 0.0003 & 0.0002 & 0.0008 & 0.0005 & 0.0003 \\
\hline $\mathrm{a}$ & 0.0018 & 0.0103 & 0.0001 & 0.0013 & 0.0016 & 0.0016 \\
\hline $\mathrm{b}$ & 0.0090 & 0.0143 & 0.0063 & 0.0050 & 0.0025 & 0.0015 \\
\hline c & 0.0065 & 0.0100 & $0 . \overline{0056}$ & 0.0050 & 0.0026 & 0.0013 \\
\hline $\mathrm{f}$ & 0.0066 & 0.0133 & 0.0060 & 0.0048 & 0.0012 & 0.0000 \\
\hline e & 0.0113 & 0.0133 & 0.0091 & 0.0048 & 0.0011 & 0.0002 \\
\hline
\end{tabular}

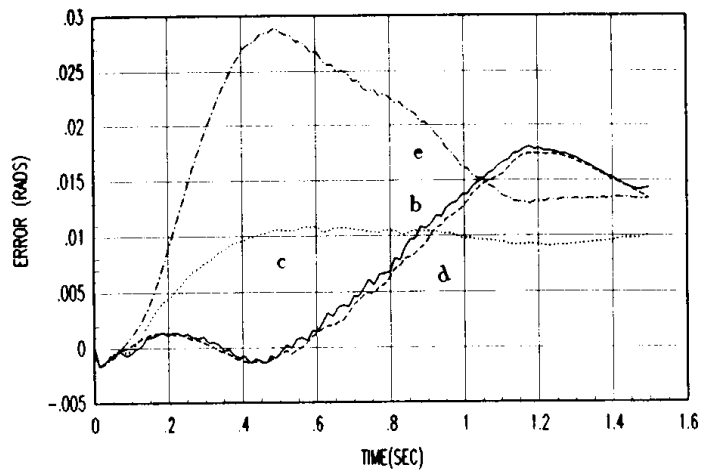

Figure 1 Joint 2 Unloaded Tracking Accuracy

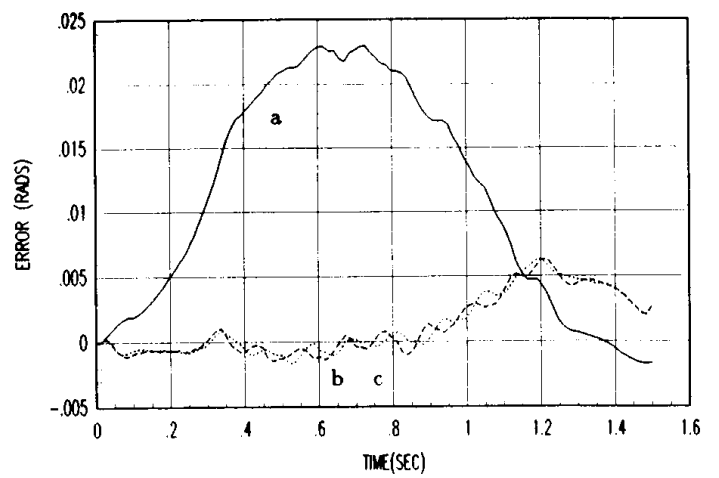

Figure 2 Joint 5 Unloaded Tracking Accuracy

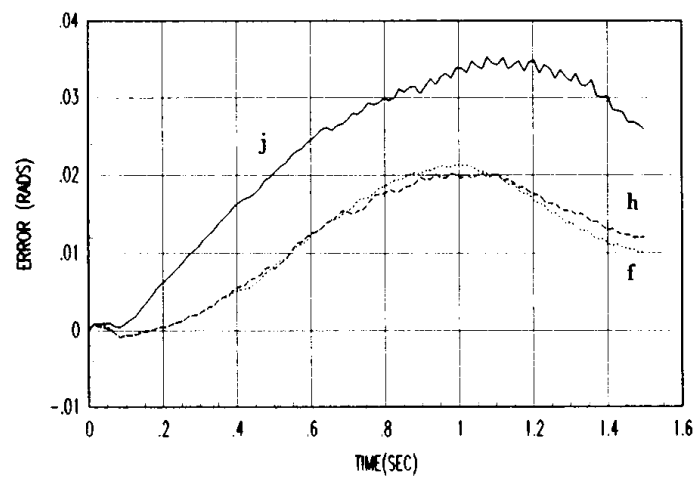

Figure 3 Joint 2 Loaded Tracking Accuracy

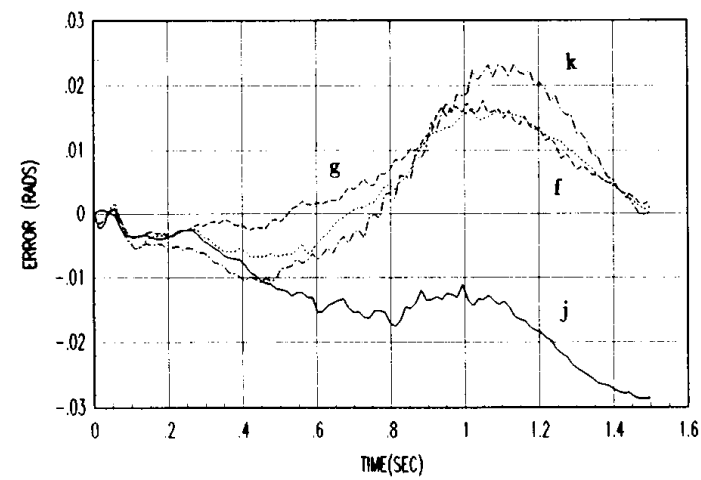

Figure 4 Joint 5 Loaded Tracking Accuracy

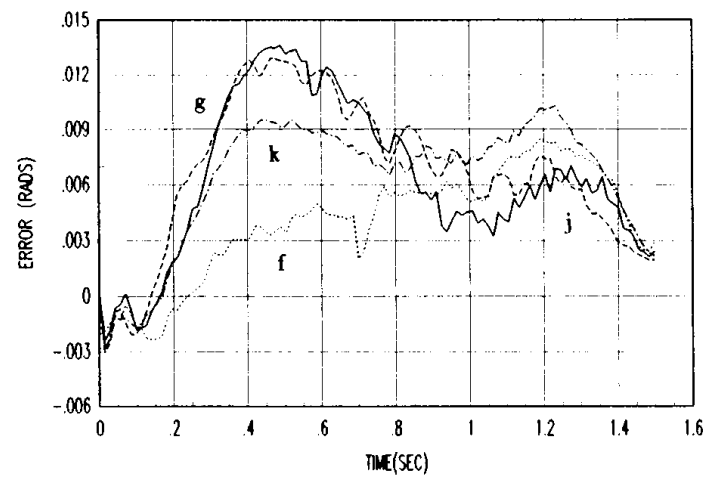

Figure 5 Joint 6 Loaded Tracking Accuracy

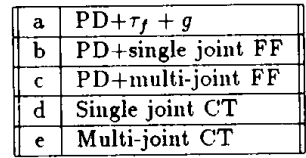

\begin{tabular}{||c|l||}
\hline$f$ & FC/FCPD with complete knowledge of payload dynamics \\
\hline$g$ & FC/FCPD with mass and centroid information \\
\hline $\bar{h}$ & FC/FCPD with mass information \\
\hline $\mathbf{j}$ & FC/FCPD with no payload information \\
\hline $\mathbf{k}$ & $\mathbf{f}$ without inertial coupling \\
\hline
\end{tabular} 